\title{
81
}

\section{THE EVOLUTION OF DOMINANCE; REPLY TO PROFESSOR SEWALL WEIGHT}

IN a thoughtful note in the AMERICAN NATURALIST of May-June, 1929, entitled "Fisher's Theory of Dominance," Professor Sewall Wright gives the results of his calculations as to the intensity of the selective agency to which I called attention, and "reaches a figure so 'extremely slow' as to make its efficacy seem highly questionable."

Since Professor Wright has carried out independently some of the calculations given in my original paper (April, 1928), and expresses the results in a different notation, it is perhaps worth while to point out that the numerical values of his primary formulas differ in no essential respects from my own, and that the selective intensity which inclines Professor Wright to reject the theory is in fact the same that originally led me to adopt it.

Professor Wright denotes the frequency of the mutant gene by 1 - $\mathrm{q}$, in place of $\mathrm{p}$; the mutation rate by $\mathrm{u}$ in place of $\mathrm{k}$, and the selective disadvantage of the heterozygote by hs in place of $1-v$, and arrives at an equation for equilibrium in the form

$$
q=1-\frac{u}{h s}
$$

in place of the equivalent equation

$$
p(1-v)=k
$$

of my paper.

At this point a slight divergence occurs between Professor Wright's method and mine, for he proceeds to calculate the intensity of the selection acting upon a single hypothetical modifying factor, whereas my method is to calculate the fraction which the heterozygotes, in which the modifying factors are exposed to selection, will contribute to the ancestry of future generations. Finding that this fraction may on quite reasonable assumptions be as high as one in 5,000, though in other cases it may be much lower, and believing that the number of generations which have occurred in the course of evolution has been more like a million times the number of generations in our experiments, I was led to conclude that this cause of modification could not safely be neglected. 
Professor Wright, on the other hand, naturally finds that the intensity of selection is of the same order as the frequency of mutations of the primary factor, and one is only surprised that this fact seems to him to settle the question. That it does so seem is partly due to the fact that Professor Wright has intended to introduce assumptions as favorable as possible to my views. He assumes a single dominant modifier, the effect of which is to cause complete dominance of the wild type gene in the primary factor. The rate of change per generation in the frequency, p' (Professor Wright uses p), of this modifier is approximately calculated, and it is shown that this rate of change, termed "the pressure per generation towards its fixation," is never more than half the mutation rate, k; actually on Professor Wright's formula it never exceeds $8 \mathrm{k} / 27$, which maximum value it attains at $\mathrm{p}^{\prime}=2 / 3$.

In case any one should he tempted to quote this result, I ought to point out that it is wrong, in that no account is taken of the effect of the frequency p' of modifying genes on the frequency of the heterozygotes which they modify, and secondly that the rate of change of gene frequency is not a direct measure of the selective intensity, such as Professor Wright requires in the more important difficulty, which he develops later. The rate of change per generation in the gene ratio $\mathrm{p}^{\prime}$ is related to the selective intensity in its favor, which we may call $i$, by the equation

$$
8 \mathrm{p}^{\prime}=\mathrm{p}^{\prime}\left(\mathrm{I}-\mathrm{p}^{\prime}\right) \mathrm{i},
$$

and it is this selective intensity, and not $\delta \mathrm{p}^{\prime}$, which must be compared with any other selective ageneies capable of modifying the gene ratio.

In Professor Wright's calculations, the result for selective intensity is that

$$
\mathrm{i}=2 \mathrm{k}\left(1-\mathrm{p}^{\prime}\right)
$$

a quantity which never exceeds twice the mutation rate, $\mathrm{k}$, and decreases progressively as $\mathrm{p}^{\prime}$ is increased. If, using similarly approximate methods, allowance is made for the effect of modifying genes, in increasing the proportion of heterozygotes to be modified, we find

$$
i=\frac{2 k}{1-p^{\prime}}
$$

which, having the same initial value, increases without limit as $\mathrm{p}^{\prime}$ increases towards unity. The case, of course, is a highly artificial one, and chosen by Professor Wright as especially favorable 
to my theory; it Is only for this reason that it is worth while showing that a more exact calculation leads to a conclusion so different from that drawn by him. I do not in the least wish to dispute that the selective intensity will be proportional to, and generally of the order of, the mutation rate, $\mathrm{k}$, though the fact that the evolution of dominance by selection proceeds with increasing speed as dominance becomes more complete is an essential point stressed in my original note.

The real difficulty, however, felt by Professor Wright is not so simple as that the selective action upon dominance modifiers is so small that there has not been time for it to have had any appreciable effect. He makes no mention of the time probably available for these changes, in the course of evolution, and agrees with me as to the probability of demonstrating the efficacy of such selection experimentally. Where he really differs from me is in my assumption that a small selective intensity of say $1 / 50,000$ the magnitude of a larger one will produce the same effect in 50,000 times the time. He suggests that the gene ratio of the modifying factors will either be held in stable equilibrium by more powerful forces, so that a minute selective intensity will merely shift to a minute extent the position of equilibrium, and produce no progressive effect - as if the complex of gene ratios were a gel rather than a sol-or be irresistibly increased or decreased by selective agencies so powerful that a minute additional selective intensity can only delay or accelerate the extinction of the less favored gene, without ever being able to determine which gene shall be extinguished-much as a wind blowing along a railroad will not exert any effect in accumulating rolling stock at the leeward terminal.

This is a criticism genuinely aimed at my theory; I do make the assumption, and the assumption really may be doubted. It is a well-directed criticism, but not a very strong one. Professor Wright can not say, "Certain observational facts do seem to support your theory, but your conjectural cause is demonstrably too feeble to have brought about its supposed effects," unless he adds, "provided I am right in my own conjecture that small selective intensities do not have effects proportional to their magnitude." My theory, in fact, is opposed, not by an obstinate fact of arithmetic, but by a rather original conjecture as to the inefficacy of minute selections, or as to the agencies controlling the gene ratios of modifying factors. 
There seems little probability of direct evidence upon so subtle a question. I have myself attached a good deal of importance, in other connections, to gene ratios held in stable equilibrium, though it never occurred to me that such a state was universal. Even if it were nearly so, the conditions on which the stability rests must be liable to change; stability may turn to instability, a transient state, admittedly, but one in which a minute, but steadily increasing, selective intensity would be well fitted to tip the balance. As to the ratios having neutral stability, there is one reason for thinking that the factors suffering the feeblest selective action will at any one time be the most numerous. The fate of those powerfully selected is quickly settled; they do not long contribute to the variance. It is the idlers that make the crowd, and very slight attractions may determine their drift. On the whole, it seems that the most reasonable assumption which we can make, on an obscure subject, is that the effect is approximately proportional to the cause.

Any progressive change, which is very slow in its action, may be represented, for the sake of paradox, as dependent upon very rare, and therefore very improbable events. In the course of time the height of a mountain is lowered by 1,000 feet; at one instant its height is above 10,000 feet, a second later it is below. What an improbable event! That the topmost grain should just protrude a fraction of a millimeter above 10,000 feet, and then that some purely conjectural meteorological accident should remove it. How can any one take seriously such an extravagant series of assumptions ? Equally improbable seems the extinction or "fixation" of modifying genes, a very rare, but occasionally necessary, consequence of the slight constant tendencies at work in modifying their frequencies.

ROTHAMSTED EXPERIMENTAL STATION,

R. A. FISHER

HARPENDEN, ENGLAND 